\title{
Giant Rectal Gastrointestinal Stromal Tumors: A Report of Two Cases
}

\section{Dickhoff ${ }^{a} \quad$ R.J. Leguit ${ }^{b} \quad$ J.F.M. Slors ${ }^{a} \quad$ W.L. Vervenne ${ }^{c}$}

W.A. Bemelman ${ }^{\text {a }}$

Departments of a Surgery, ${ }^{b}$ Pathology and 'Oncology, Academical Medical Center, University Hospital, Amsterdam, The Netherlands

\section{Key Words}

Gastrointestinal stromal tumor · Imatinib $\cdot$ Rectal $\cdot$ Neo-adjuvant

\begin{abstract}
Giant gastrointestinal stromal tumors (GISTs) of the rectum are rare and often difficult to remove surgically. At the time metastases are found, GISTs are considered to be incurable and until recently no adequate therapy was of any value for these patients. Recently, imatinib was introduced: a signal transducing inhibitor acting specifically on the KIT-tyrosine kinase, which can be used to downsize giant GIST (neo-adjuvant) before surgery or induce stable disease in case of metastases with few minor side-effects. Two patients with giant rectal GIST are presented, one of which was treated before the imatinib era, the other when imatinib was available.
\end{abstract}

\section{Introduction}

Gastrointestinal stromal tumors (GISTs) are rare tumors, but still are the most common mesenchymal tumors of the gastrointestinal tract (approximately $80 \%$ ). Mostly they occur in the stomach (60-70\%) and in the small bowel (20-30\%). Localisation in the esophagus is rare $(<5 \%)$ and they are found in the colon and rectum in approximately $5-$ $10 \%$ of cases $[1-3]$.

GISTs can become very large, and when their size reaches over $10 \mathrm{~cm}$, they are referred to as 'giant GIST'. These giant types may occur everywhere in the gastrointestinal tract but will grow to these sizes especially in those regions where they may cause the least symptoms at a certain size. Rectal obstruction is a late symptom, as duodenal obstruction might become symptomatic already with a smaller diameter of the tumor. Therefore, clinical presentation mainly depends on tumor size and localisation or is determined by metastatic disease or in-growth in adjacent organs. 
The most frequent complaint of patients with a rectal GIST is change in bowel movement due to tumor size and/or gastrointestinal bleeding, when the overlying mucosa is ulcerated. Other symptoms are abdominal discomfort and urinary disorders by compression of the bladder.

Surgical removal remains the only curative treatment for patients with GIST [5], but tumors with metastasis are considered to be inoperable. Chemotherapy and/or radiotherapy showed to have disappointing results $[1,6]$ and no remarkable response may be expected from neither of these two.

Recently, a new drug, imatinib (STI-571), was launched, and many promising results already have been reported. Imatinib is a signal transduction inhibitor, inhibiting amongst others the signalling of the KIT-tyrosine kinase, which switches the balance towards reduced cellular proliferation and increased apoptosis [4]. Good results have been reported in the treatment of locally irresectable or metastatic GIST, in terms of partial response or stable disease $[4,7,8]$. The effect of imatinib as (neo-)adjuvant is further being investigated. Bümming et al. have reported some promising results [7], but the effect of imatinib as adjuvant and neo-adjuvant needs further investigation in prospective randomised clinical trials.

This case report is the first report of two patients treated for a giant rectal GIST, one patient with a giant GIST treated before the introduction of imatinib, the other one treated after the availability of imatinib.

\section{Cases}

\section{Case 1}

A 65-year-old woman, a Jehova's Witness, attended our hospital with vague abdominal pain, urge and occasional rectal bleeding. There was no change in bowel movement and her weight was stable. Her medical history reported hypertension and a hiatal hernia. Physical examination showed an obese (105 $\mathrm{kg}, 173 \mathrm{~cm}$ ) vital woman. Rectal examination showed a large palpable mass located posteriorly close to the pelvic floor. At sigmoidoscopy, a giant ulcerating tumor was seen from 2 to $18 \mathrm{~cm}$ in the posterior wall of the rectum. The central ulceration was probably caused by tumor compression of the rectum. Abdominal CT and MRI demonstrated a large tumor of at least $10 \mathrm{~cm}$ cross-section on the dorsal side of the rectal wall without invasion or ingrowth in adjacent organs, but with intralesional bleeding (fig. 1). Ultrasonography of the liver and chest X-ray showed no lesions suspect for metastases.

At this point the diagnosis was a mesenchymal tumor. Since she was bleeding persistently, and radiotherapy and chemotherapy did not seem a valid option for this kind of tumor, the patient went for surgery. After hemodilution and using the cell saver, an abdominoperineal resection of the rectum with colostoma was performed. A sacral plexus bleeding complicated surgery. Since it was not permitted to administer blood products, the pelvis was packed at a hemoglobin level of $2 \mathrm{mmol} / \mathrm{l}$. The gauze were left in for a week, and removed when the hemoglobin level was $3 \mathrm{mmol} / \mathrm{l}$. Her further recovery was uneventfull.

Pathological examination of the resected specimen showed a GIST of the rectal wall with diffuse Ckit positivity. The high mitotic rate $\left(18\right.$ mitotic figures per $\left.2 \mathrm{~mm}^{2}\right)$ and the large tumor size $(15 \mathrm{~cm})$ indicate high malignant potential. The capsule was ruptured so that complete removal of the tumor could not be ensured.

Approximately one year after resection of the primary tumor, a hypodense lesion was found in the liver with routine ultrasound. The subsequent CT scan showed recurrent disease in the iliac fossa and in the liver. At that time imatinib was available and administered in a daily dose of $400 \mathrm{mg}$. As a sign of response the metastases typically became clearer at CT follow-up. Until now, almost three years after initiation of imatinib therapy, she has stable disease. 
Case 2

A 62-year-old man was referred to the surgeon and urologist because of progressive obstipation, painful defecation and voiding problems. In the past 6 months he lost $17 \mathrm{~kg}$ in weight. Physical examination showed a jaundiced man. The liver was not palpable. Rectal examination revealed a palpable tumor, $5 \mathrm{~cm}$ proximal to the dentate line.

Blood tests showed elevated liver enzymes and bilirubin. Endosonography of the rectum demonstrated a submucosal tumor. A CT scan of the abdomen showed a large extraluminal tumor in the rectum compressing the lumen and multiple hypodense lesions in the liver, suspect for being metastases. One of these lesions counted $8.9 \times 8.6 \mathrm{~cm}$ and one was compressing the bile system. Furthermore, there was a solitary lung lesion suspected for lung metastasis.

Ultrasound-guided needle biopsy of the liver lesions showed a tumor composed of polymorphic spindle cells with a positive C-kit immunohistochemical staining, infiltrating pre-existent liver parenchyma (fig. 2). Furthermore these lesions showed necrosis and a high mitotic rate, consistent with a metastasis of a malignant GIST.

Imatinib at a dose of $400 \mathrm{mg}$ once daily was initiated. Within a couple of weeks his symptoms of obstruction, voiding problems and disturbed liver function decreased. Follow-up CT scan showed regression and clarification of the GIST and metastases as a sign of response ( $\underline{\text { fig. } 3 \text { ). }}$.

\section{Discussion}

Until recently, the prognosis of patients with a giant GIST of the rectum was very poor, especially when metastases were present at time of diagnosis. Radiotherapy and chemotherapy are not effective in treating these tumors or metastasis and surgery used to be the only therapeutical option for non-metastatic disease [1]. The development of the new drug imatinib (Glivec ${ }^{\mathrm{TM}}$ ) improved the therapeutic options tremendously. Where giant GIST used to be irresectable and metastatic disease uncontrollable, imatinib offers the opportunity of tumor regression and stable disease. In the EORTC study for GISTs, complete response was $7 \%$, partial response $25 \%$, stable disease was reached in $24 \%$ of the cases, and in $14 \%$ there was no effect and disease progressed after starting imatinib treatment.

Long-term follow-up must be awaited to clarify the behavior of the tumor and metastases on long-term treatment with this new tyrosine kinase inhibitor. Verweij et al. found that to achieve response induction, a daily dose of $400 \mathrm{mg}$ is sufficient to reach this goal, but a dose of $400 \mathrm{mg}$ twice a day achieves significantly longer progression-free survival, especially in widespread metastatic disease [9].

Imatinib as a neo-adjuvant to downsize giant GIST of the rectum before surgery might help reach resectability of the tumor. At the time the first patient, case 1, attended our hospital, the drug was not available for clinical use in The Netherlands. At present, first a treatment with imatinib would be initiated to downsize the tumor in order to improve the likelihood of a radical resection and reduce perioperative morbidity. Surgery remains the only chance of complete removal of the GIST. As an adjuvant, imatinib is promising in reaching stable disease and prolong symptom-free interval after surgery, especially in metastatic disease or when local recurrence occurs [10].

At the time of diagnosis approximately $10-50 \%$ of patients have overtly metastatic disease [4]. For large irresectable symptomatic gastrointestinal tumors of the rectum with metastatic spread, single therapy with this drug might also be an option to decrease symptoms and even no surgery will be necessary in some cases. This is shown in case 2, where stable disease was reached and symptoms disappeared after starting imatinib. For 


\begin{tabular}{c|l|l|l}
$\begin{array}{c}\text { Case Reports in } \\
\text { Gastroenterology }\end{array}$ & $\begin{array}{l}\text { Case Rep Gastroenterol 2008;2:54-59 } \\
\text { D0I: 10.1159/000119317 }\end{array}$ & Published online: March 11, 2008 & $\begin{array}{l}\text { O 2008 S. Karger AG, Basel } \\
\text { ISSN 1662-0631 } \\
\text { www.karger.com/crg }\end{array}$ \\
\hline
\end{tabular}

now, long-term follow-up and prospective trials are needed to explore the possibilities of this novel drug which is a breakthrough in the treatment of a potentially lethal disease.

Fig. 1. MRI scan showing a large mass in the lower abdomen and pelvis, with intralesional bleeding.

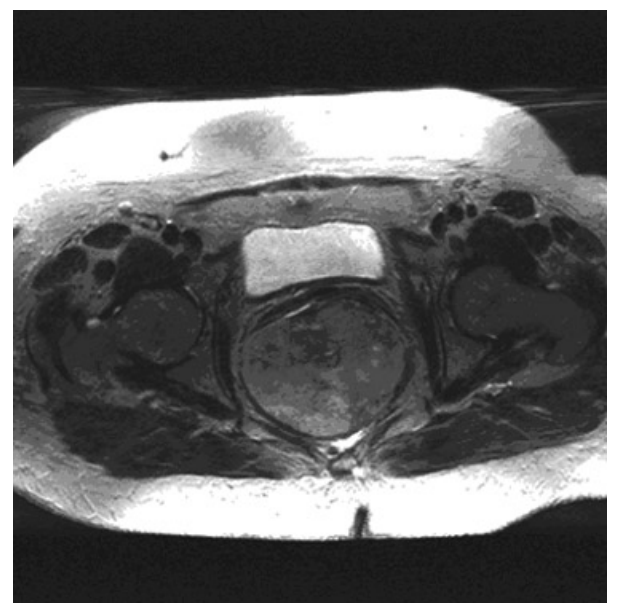

Fig. 2. Gastrointestinal stromal tumor (GIST). a A polymorphic spindle cell tumor infiltrating preexistent liver parenchyma (right side). Hematoxylin and eosin; original magnification $\times 200$. $\mathbf{b}$ The tumor shows diffuse c-kit positivity. C-kit; original magnification $\times 100$.

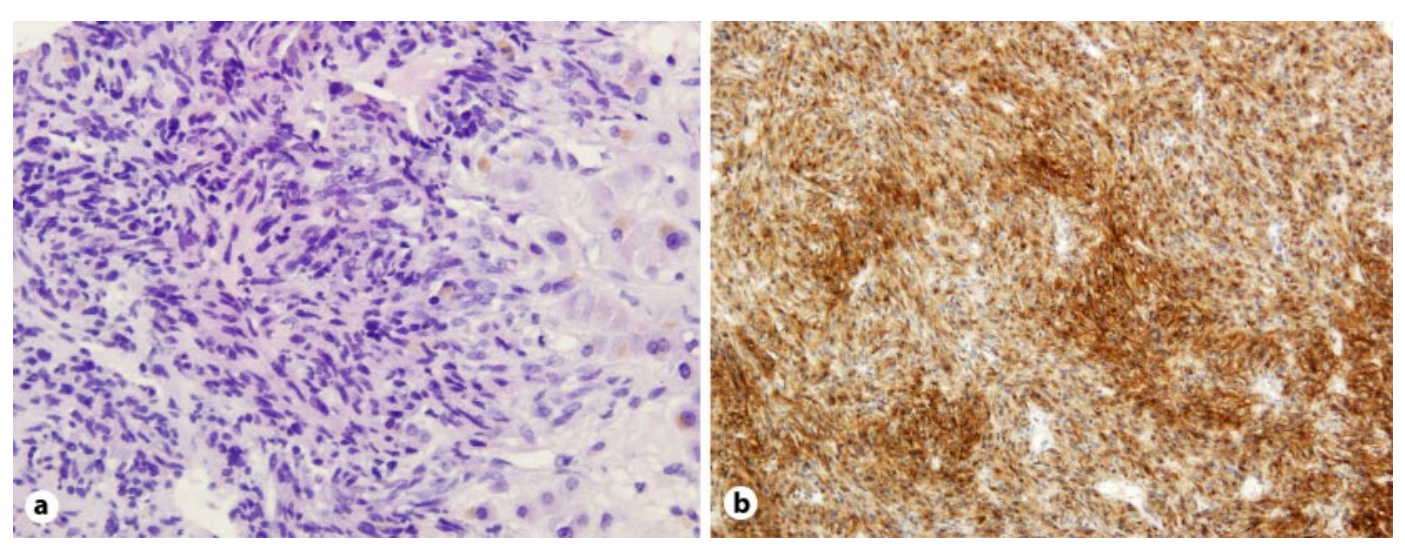


Fig. 3. CT scan showing both regression of tumor (a) and metastasis (c) after 3 months of Glivec therapy $(\mathbf{b}, \mathbf{d})$.
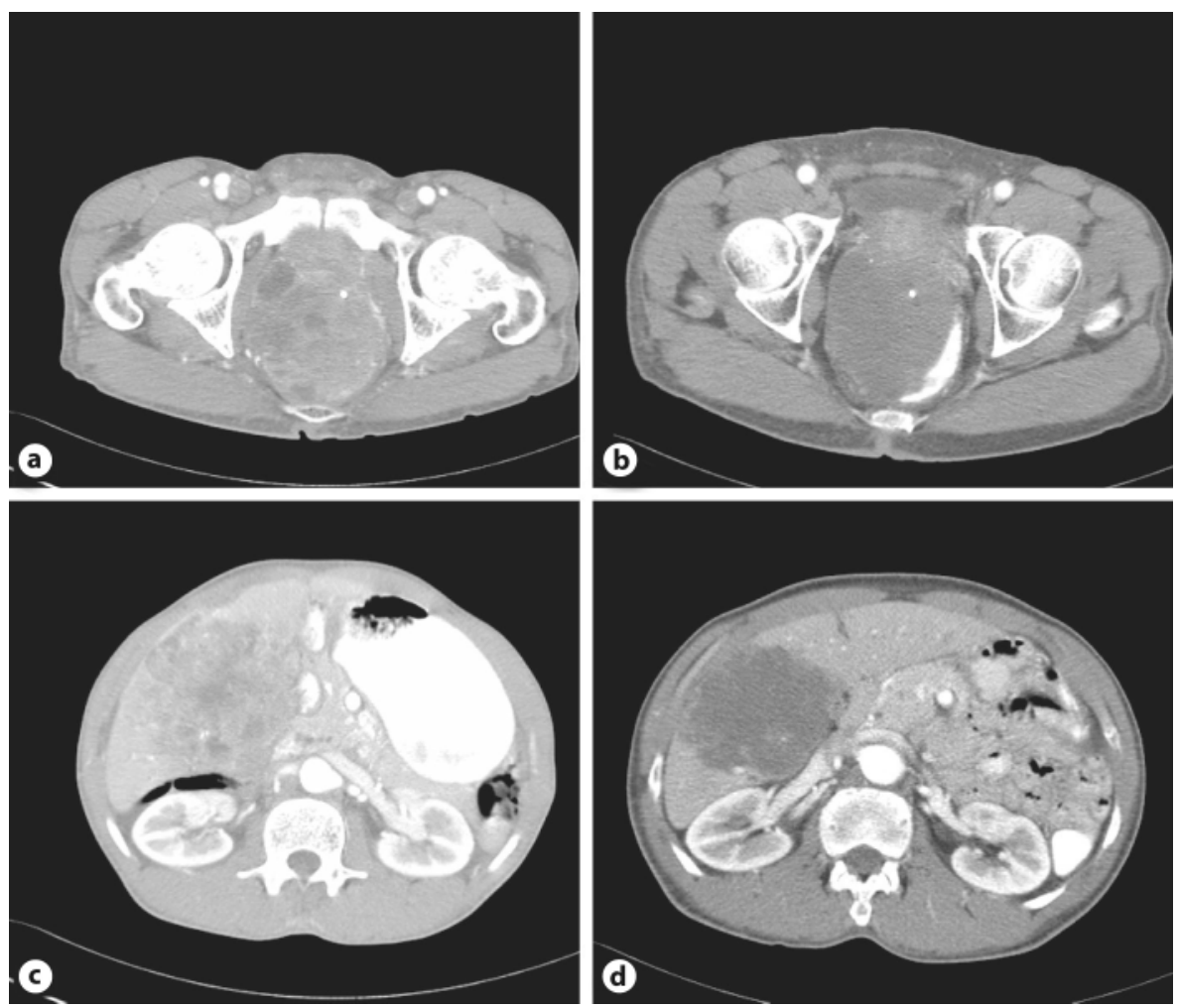


\section{References}

1 DeMatteo RP, Lewis JJ, Leung D, Mudan SS, Woodruff JM, Brennan MF: Two hundred gastrointestinal stromal tumors: recurrence pattern and prognostic factors for survival. Ann Surg 2000;231:51-58.

-2 Joensuu H, Fletcher C, Dimitrijevic S, Silberman S, Roberts P, Demetri R: Management of malignant gastrointestinal stromal tumours. Lancet Oncol 2002;3:655-664.

-3 Miettinen M, Lasota J: Gastrointestinal stromal tumors - definition, clinical, histological, immunohistochemical, and molecular genetic features and differential diagnosis. Virchows Arch 2001;438:1-12.

4 Conolly EM, Gaffney E, Reynolds JV: Gastrointestinal stromal tumours. Br J Surg 2003;90:1178-1186.

5 Miettinen M, El-Rifai W, Sobin LH, Lasota J: Evaluation of malignancy and prognosis of gastrointestinal stromal tumors: a review. Hum Pathol 2002;33:478483.

6 Bucher P, Villiger P, Egger JF, Buhler LH, Morel P: Management of gastrointestinal stromal tumours: from diagnosis to treatment. Swiss Med Wkly 2004;134:145-153.

7 Bümming P, Andersson J, Meis-Kindblom JM, Klingenstierma H, Engström K, Stierner U, Wängberg B, Jansson S, Ahlman H, Kindblom LG, Nilsson B: Neoadjuvant, adjuvant and palliative treatment of gastrointestinal stromal tumours (GIST) with imatinib: a centre-based study of 17 patients. Br J Cancer 2003;89:460-464.

8 Gelderblom H, Verweij J: Enkele patienten met een gemetastaseerde gastrointestinale stromaceltumor: gekeerde kansen door de introductie van imatinib. Ned Tijschr Geneeskd 2003;147:2041-2043.

-9 Verweij J, Casali PG, Zalcberg J, LeCesne A, Reichardt P, Blay JY, Issels R, van Oosterom A, Hogendoorn PCW, van Glabbeke M, Bertulli R, Judson I: Progression-free survival in gastrointestinal stromal tumours with high-dose imatinib: randomised trial. Lancet 2004;364:1127-1134.

10 Chen TW, Liu HD, Shyu RY, Yu JC, Shih ML, Chang TM, Hsieh CB: Giant malignant gastrointestinal stromal tumors: Recurrence and effects of treatment with STI-571. World J Gastroenterol 2005;11:260-263. 